\title{
A Polymorphic Intermediate Verification Language: Design and Logical Encoding
}

\author{
K. Rustan M. Leino ${ }^{1}$ and Philipp Rümmer ${ }^{2}$ \\ 1 Microsoft Research, Redmond \\ leino@microsoft.com \\ 2 Oxford University Computing Laboratory \\ philr@comlab.ox.ac.uk
}

\begin{abstract}
Intermediate languages are a paradigm to separate concerns in software verification systems when bridging the gap between programming languages and the logics understood by theorem provers. While such intermediate languages traditionally only offer rather simple type systems, this paper argues that it is both advantageous and feasible to integrate richer type systems with features like (higher-ranked) polymorphism and quantification over types. As a concrete solution, the paper presents the type system of Boogie 2, an intermediate verification language that is used in several program verifiers. The paper gives two encodings of types and formulae in simply typed logic such that SMT solvers and other theorem provers can be used to discharge verification conditions.
\end{abstract}

\section{Introduction}

Building a program verifier is a complex task that requires understanding of many domains. Designing its foundation draws from domains like semantics, specifications, and decision procedures, and constructing its implementation involves knowledge of compilers and software engineering. The task can be made manageable by breaking it into smaller pieces, each of which is simpler to understand. A successful practice (e.g., [1145]) is to make use of an intermediate verification language [16 110].

The intermediate verification language serves as a thinking tool in the design of the verifier front end for each particular source language. As such, it must provide a level of abstraction that is high enough to give leverage to the front end. At the same time, there is a risk that the general translations of higher-leverage features become too cumbersome to sustain good decision procedure performance. Some higher-leverage features, like a fancy type system, provide safety to the front end by restricting what intermediate programs are admissible. At the same time, there is a risk that such restrictions lead to cumbersome encodings in the front end, especially compared to the encodings that are possible by directly using the more coarse-grained type system of a decision procedure.

In this paper, we introduce the type system of the intermediate verification language Boogie 2 developed by the authors, the successor of BoogiePL [81]. Unlike its untyped predecessor, whose type annotations were mainly used for some consistency checks, Boogie 2 features an actual type system. Going beyond the Hindley-Milner style types in the intermediate verification language Why [10], Boogie 2 features polymorphic

J. Esparza and R. Majumdar (Eds.): TACAS 2010, LNCS 6015, pp. 312-327, 2010.
(C) Springer-Verlag Berlin Heidelberg 2010 
maps, higher-rank polymorphism, and impredicativity, which are useful in modeling the semantics of a type-safe object store (as in Spec\# or Java).

In addition to introducing the polymorphic features of Boogie, we describe our translation of Boogie's polymorphic logic into simply typed logic, which is used by many satisfiability modulo theories (SMT) solvers that support the SMT-LIB format [2]. In fact, we give two different translations into simply typed logic, and we present performance figures from substantial benchmarks that compare these. The benchmarks come from the Spec\# program verifier [1], the VCC [5] and HAVOC [4] verifiers for C, and Dafny [14], all of which build on Boogie. All of the benchmarks make extensive use of so-called triggers required for e-matching [9], and our experiments give evidence to that the triggers are properly maintained by our translations.

The contributions of our work are: (i) An impredicative type system for an intermediate verification language, featuring full higher-ranked polymorphism, (ii) two translations of the verification language, and especially its polymorphic maps, into simply typed logic suitable for SMT solvers, (iii) experimental data comparing the performance of the two translations with each other and with an (unsound) translation ignoring types.

\section{Boogie 2 Types and Expressions}

A Boogie program consists of a set of mathematical and imperative declarations that define a set of execution sequences. The Boogie program is correct if none of those execution sequences contains an error state [13]. Programs can be written by hand, but most Boogie programs are machine generated by various program verifiers to encode the semantics of given source programs. For example, the source-language declaration in Fig. 1 can be modeled in Boogie as shown in Fig. 2, where the object store is represented explicitly by a variable Heap whose type is a map from object references and field names to values (we explain this example in more detail later).

For the purposes of this paper, one can think of the imperative features of Boogie as convenient syntactic shorthands for writing Boogie expressions. Hence, we focus on Boogie's expressions and their types. For further details of the language, we refer to the Boogie 2 language reference manual [13].

\subsection{Type Declarations}

The built-in types of Boogie are booleans (bool), mathematical integers (int), and bit-vector types of every size ( bv0, bv1, bv2, . ). In addition, there are map types, which we describe below, and user-defined type constructors. A program can also declare parameterized type synonyms, which are essentially like macros, thus providing syntactic convenience but not adding to the expressiveness of the type system. A type denotes a nonempty set of individuals, and the sets denoted by different types are disjoint. Each different parameterization of a type constructor yields a distinct type, each denoting an uninterpreted set of individuals. For example, the type declarations in Fig.2 introduce a nullary type constructor Ref and a unary type constructor Field. The sets of individuals denoted by Ref, Field int, and Field Ref are all disjoint. 


\section{class Person \{ int age; bool isMarried; \}}

Fig. 1. An example code snippet from a source program

type Ref;

type Field $\alpha$;

type Heap Type $=\langle\alpha\rangle[$ Ref , Field $\alpha] \alpha$; const unique age: Field int; const unique isMarried: Field bool; var Heap: Heap Type;

function Is WellFormed (Heap Type) returns (bool);

const unique snapshot: Field Heap Type;

Fig. 2. An example of how object-oriented program features, like those in Fig. 1, can be modeled in Boogie (the language features used are introduced in detail in Section 2. Ref is a type and Field is a unary type constructor. Type synonym Heap Type is defined as the polymorphic map type that represents the heap. Is WellFormed demonstrates that functions can take polymorphic maps as arguments. For any $r$ of type Ref, Heap $[r$, snapshot] has type Heap Type, illustrating that polymorphic maps can be arbitrarily nested (an instance of impredicativity). The modifier unique is used to say that the constant declared has a different value than all other unique constants, which for the 3 constants here also follows from the fact that their types are different.

\subsection{Expressions}

Boogie expressions include variables and constants, function applications, logical, arithmetic, and relational operators, as well as logical quantifiers, type coercions, and map operations. All expressions are total: every well-typed expression yields some appropriately typed value that is a function of its subexpressions. For the most part, typing of expressions is obvious and straightforward. Let us describe the more salient features.

Polymorphic Functions, Quantifications over Types. Functions can be polymorphic, that is, they can take type parameters. Analogously, the bound variables in universal and existential quantifiers can range over both individuals (of specified types) and types. Polymorphism is useful because it allows a user to provide an axiomatization of, say, pairs that is independent of the pair element types, while maintaining the type guarantee that different types of pairs are not mixed up.

For example, Fig. 3 declares a binary type constructor Pair, along with a function Cons for constructing a pair and a function Left that extracts the left element of a pair. Type parameters and bound type variables are introduced inside angle brackets, like in C\# or Java. A function declaration in Boogie only defines the signature of the function; properties of functions can be defined by axioms. The figure includes an axiom that defines the relationship between Cons and Left. Note that the quantification is over any element types $\alpha$ and $\beta$ and any elements $a$ and $b$ of those types. Hence, the axiom applies generically to pairs with any element types.

The meaning of a function depends on its type-parameter instantiation. That is, a polymorphic function $f$ is really a family of functions $\bar{f}$, one for each possible instantiation (e.g., fint, $\left.f_{\text {Ref }}\right)$.

Type Coercions. Boogie infers instantiations for type parameters of function applications. Usually, they can be inferred from the types of the function's arguments, but 


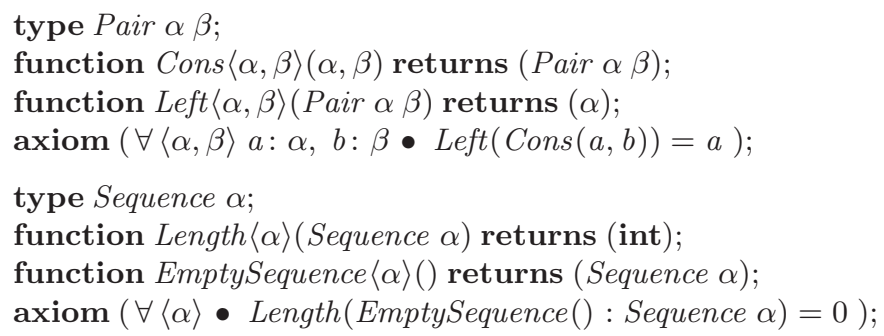

Fig. 3. Examples of polymorphic functions and quantifications over types in Boogie. In the last line, the quantifier ranges only over types, not over any individuals, and the type coercion makes the application EmptySequence() well-typed.

sometimes it is also necessary to consider the context of the function application. In particular, if a type parameter is used among the domain types in the function's signature, then its instantiation in a function application can be inferred from the arguments. But in the case that a type parameter is used only in the return type, then type inference needs to consult the context. Type parameters that are not used in either the domain types or the result type are not allowed.

For example, Fig. 3 declares a function that gives the length of a generic sequence. Function EmptySequence returns a zero-length sequence of any type. Type parameter $\alpha$ is used only in the return type of EmptySequence, which is common and useful for this and similar functions. Hence, to infer the type parameter in an application EmptySequence(), the context surrounding the application must be used.

An error is reported if an instantiation for type parameters cannot be determined uniquely. To deal with such cases, the language offers a type coercion expression $e: t$, which has type $t$, provided $t$ is a possible typing for expression $e$. For example, the expression Length(EmptySequence()) is ill-formed because of the ambiguous typeparameter instantiation; but with the type coercion in Fig. 3, the ambiguity is resolved.

Because the meaning of a polymorphic function is really that of a family of functions, note that EmptySequence int $_{\text {( }}()$ has a different value than EmptySequence Ref $_{(}()$.

Maps. In addition to functions, Boogie offers maps. Like functions, maps have a list of domain types and a result type and can be polymorphic. The difference is that maps are themselves expressions (they are "first class"), unlike functions, which can appear in an expression only when applied to arguments. This means that program variables can hold maps (like Heap in Fig. 2).

Though they may have the appearance of higher-order values, maps are but first-order individuals, and to "apply" them to arguments, one applies Boogie's built-in map-select operator, written with square brackets (to be suggestive of retrieving an element at a given index of an array) [19]. For example, if $m$ is a map of type [int, bool] Ref, that is, a map type with domain types int and bool and result type Ref, then the expression $m[5$, false $]$ denotes a value of type Ref. Due to maps, Boogie can in many situations be used like a higher-order language (where functions can be passed around as values), but still allows the use of efficient first-order reasoners. 
If $m$ is an expression denoting a map, $i$ is a list of expressions whose types correspond to the domain types of $m$, and $x$ is an expression of the result type of $m$, then the map-update expression $m[i:=x]$ denotes the map that is like $m$, except that it maps $i$ to $x$ [19]. Using common notation for arrays, the imperative part of Boogie allows the assignment statement $m[i]:=x$; as a shorthand for $m:=m[i:=x]$;.

Boogie does not promise extensionality of maps, that is, the property that maps with all the same elements are equal; for example, $m$ and $m[i:=m[i]]$ are not provably equal, but they are provably equal at all values of the domains. From our experience, extensionality is not required for most applications; the motivation to exclude extensionality by default is the better performance of decision procedures for non-extensional maps. Where extensionality is needed, users can supply the required axioms themselves.

A novel and key feature of maps in Boogie is that they can be polymorphic. To motivate this feature, let us consider one of the most important modeling decisions that the designer of a program verifier faces: how to model the memory operated on by the source language. For example, for a type-safe object-oriented language, one may choose to model the object store (the heap) as a two-dimensional map from object references and field names to values [23 1 14]. Since the result type of such a map depends on the selected field name, it is natural to declare the heap to be of a polymorphic map type. (Without polymorphic maps, one either needs to introduce explicit cast functions or split the one heap variable into several.)

As we already alluded to, Fig. 2 shows by example some Boogie declarations that a verifier might use to encode the semantics of the object-oriented program in Fig. 1 . (cf. [1]14]). In the example, Ref is used to denote the type of all object references, Field $\alpha$ denotes the type of field names that in the heap retrieve values of type $\alpha$, and $\langle\alpha\rangle[$ Ref, Field $\alpha] \alpha$ is the polymorphic map type of the heap itself. For instance, if $r$ is a reference, then Heap $[r$, age $]$ is an integer and Heap $[r$, isMarried $]$ is a boolean.

Boogie's type system allows advanced uses of polymorphic maps, which is useful for the kind of semantic models one defines in a program verifier. For example, it is common to want to define properties of heaps, for example distinguishing heaps that satisfy some sort of well-formedness condition from heaps that do not. A natural way to do that is to start by defining a function on heaps, like Is WellFormed in Fig. 2. This is an example of a higher-rank type.

Type parameters of maps are like those of functions: each type parameter must be used in either the domain types or the result type of the map type, and it is an error if type inference cannot uniquely determine the instantiations of type parameters. And as for functions, a polymorphic map is really a family of maps, one for each possible type-parameter instantiation. For example, a map $m$ of type $\langle\alpha\rangle[$ int $] \alpha$ really denotes a family of maps $\bar{m}$, and $m_{\text {int }}[E]$ has a different value than $m_{\text {bool }}[E]$. It should also be noted that the types $[\alpha] T$ and $\langle\alpha\rangle[\alpha] T$ are different: the first is a type with a free type parameter $\alpha$ and can be instantiated to any (monomorphic) map type $[s] T$, while the second describes polymorphic maps from any type to $T$.

Equality among map types does not depend on the names or order of type parameters. For example, the type $\langle\alpha, \beta\rangle[\alpha, \beta]$ int is equal to $\langle\gamma, \delta\rangle[\delta, \gamma]$ int. Polymorphism, however, is significant: the types [int] bool and $\langle\alpha\rangle[\alpha]$ bool are incompatible. 
Equality. Equality in Boogie is standard mathematical equality, but the typing of equality expressions in Boogie is more liberal than is absolutely the standard. The equality expression $E=F$ is allowed if there is some instantiation of enclosing type parameters that makes the types of $E$ and $F$ equal. Let us motivate this typing rule.

A common way to specify the effects of a source-language procedure is to use a modifies clause that lists the object-field locations in the heap that the procedure is allowed to modify. The modifies clause is then encoded into Boogie as a procedure postcondition that specifies a relation between the procedure's heap on entry, written $\operatorname{old}($ Heap), and its heap on return, written Heap (see, e.g., [14]). For instance, to encode that a procedure's effect on the heap in the source language is limited to $p$.age and $p$.isMarried, one can in Boogie use a postcondition like

$$
\begin{aligned}
(\forall\langle\alpha\rangle r: \text { Ref, } f: \text { Field } \alpha \bullet & \text { Heap }[r, f]=\operatorname{old}(\text { Heap }[r, f]) \vee \\
& (r=p \wedge f=\text { age }) \vee(r=p \wedge f=\text { isMarried }))
\end{aligned}
$$

In order to type check this expression, it is necessary for the type system to consider the possible instantiation $\alpha:=$ int for $f=$ age and $\alpha:=$ bool for $f=$ isMarried, and Boogie does exactly that. Being liberal in this typing rule does not cause any semantical problems in Boogie: because different types represent disjoint sets of individuals, an equation simply evaluates to false if the two sides of the equation evaluate to individuals of different types. For example, for the $f$ in the quantifier above, $f=$ age $\wedge f=$ isMarried type checks but always evaluates to false.

\subsection{Formalization of the Type System and Type Checking}

The abstract syntactic category of types is described by the following grammar:

$$
\text { Type }:=\alpha \mid \text { C Type } \text { T }^{*}\left\langle\alpha^{*}\right\rangle\left[\text { Type }^{*}\right] \text { Type }
$$

in which $C \in \mathcal{C}$ ranges over type constructors (with a fixed arity $\operatorname{arity}(C)$ ) and $\alpha \in \mathcal{A}$ over an infinite set of type variables. We assume that $\mathcal{C}$ always contains the pre-defined nullary constructors bool, int, bv0, bv1, bv2, ... Only those types are well-formed in which type constructors receive the correct number of argument types, and in which type parameters of polymorphic map types occur in the map domain or result types.

For two types $s, t \in$ Type, we write $s \equiv t$ iff $s$ and $t$ are equal modulo renaming or reordering of bound type parameters. A type substitution is a mapping $\sigma: \mathcal{A} \rightarrow$ Type from type variables to types. Substitutions are canonically extended on all types, assuming that variable capture is avoided by renaming bound type variables when necessary.

Formalizing the typing of expressions, the judgment $\mathcal{V} \Vdash E: t$ says that in a context with variable-type bindings $\mathcal{V}$, expression $E$ can be typed as type $t$. Figure 4 shows the most important typing rules. All other operators are typed as in the rule for function application. In the figure and the whole paper, $\mathcal{F}$ denotes the set of declared functions and constants, whereas $\mathcal{X}$ denotes an infinite set of variables.

Note that for any type-correct program, all type-parameter instantiations have been resolved. But this does not mean that the application of a polymorphic function or map can easily be replaced by a specific monomorphic instance, because of quantifications over types. For example, the application of EmptySequence in Fig. 3 is resolved to EmptySequence ${ }_{\alpha}$, but $\alpha$ is a quantified type variable that refers to any type; hence, the axiom says something about every member of the EmptySequence family. 


$$
\begin{gathered}
\frac{x \mapsto t \in \mathcal{V}}{\mathcal{V} \Vdash x: t} \quad \frac{\mathcal{V} \Vdash E: t}{\mathcal{V} \Vdash E: t: t} \\
\mathcal{V} \Vdash E: s \quad \mathcal{V} \Vdash F: t \\
\frac{\sigma(s) \equiv \sigma(t)}{\mathcal{V} \Vdash E=F: \text { bool }} \\
\mathcal{V} \Vdash m:\langle\bar{\alpha}\rangle[\bar{s}] t \\
\frac{\mathcal{V} \Vdash E_{i}: \sigma\left(s_{i}\right) \quad\left(\text { for }\left(E_{i}, s_{i}\right) \in(\bar{E}, \bar{s})\right)}{\mathcal{V} \Vdash m[\bar{E}]: \sigma(t)} *
\end{gathered}
$$

$$
\begin{aligned}
& f\langle\bar{\alpha}\rangle(\bar{s}) \text { returns }(t) \in \mathcal{F} \\
& \frac{\mathcal{V} \Vdash E_{i}: \sigma\left(s_{i}\right) \quad\left(\text { for }\left(E_{i}, s_{i}\right) \in(\bar{E}, \bar{s})\right)}{\mathcal{V} \Vdash f(\bar{E}): \sigma(t)} * \\
& \frac{(\mathcal{V}, \bar{x} \mapsto \bar{t}) \Vdash E: \text { bool } \quad Q \in\{\forall, \exists\}}{\mathcal{V} \Vdash(Q\langle\bar{\alpha}\rangle \bar{x}: \bar{t} \bullet E): \text { bool }} \\
& \mathcal{V} \Vdash m:\langle\bar{\alpha}\rangle[\bar{s}] t \quad \mathcal{V} \Vdash F: \sigma(t) \\
& \frac{\mathcal{V} \Vdash E_{i}: \sigma\left(s_{i}\right) \quad\left(\text { for }\left(E_{i}, s_{i}\right) \in(\bar{E}, \bar{s})\right)}{\mathcal{V} \Vdash m[\bar{E}:=F]:\langle\bar{\alpha}\rangle[\bar{s}] t} *
\end{aligned}
$$

Fig. 4. The typing rules for Boogie expressions. The context of type judgments is a partial mapping $\mathcal{V}: \mathcal{X} \rightarrow$ Type that assigns types to variables. The rules marked with ' $*$ ' impose the side condition $\operatorname{dom}(\sigma)=\{\bar{\alpha}\}$. The typing rules show what it means for expressions to be type correct; they abstract over how type inference is done.

\subsection{Matching Triggers}

We have one more thing to say about expressions in Boogie, and it concerns the way many SMT solvers handle universal quantifications, namely by selective instantiation. Instantiations are based on (user-supplied or inferred) matching triggers, which indicate which patterns of ground terms in the prover's state are to give rise to instantiations [9]. Boogie has support for specifying matching triggers for quantifications. For example,

$$
\operatorname{axiom}(\forall x: t \bullet\{f(x)\} f \operatorname{Inverse}(f(x))=x) \text {; }
$$

specifies the trigger $f(x)$ and says to instantiate the universally quantified variable with any value appearing among the ground terms as an argument of function $f$. In an SMT solver based solely on triggers, these are the only instantiations there will ever be. All Boogie front ends make heavy use of triggers. (For an application that uses quantifiers and an explanation of the design of triggers for that application, see [15].)

A trigger is a set of expressions, each of which will undergo the encoding into the underlying logic that we are about to describe. However, it is important that the logical encoding not interfere with user-defined triggers or automatically inferred triggers, since that might lead to poor performance (too many instantiations) or incompleteness (too few instantiations).

\section{Representation of Types as Terms}

Automated theorem provers and SMT solvers typically offer only untyped or simple multi-sorted logics as their input language (with the notable exception of Alt-Ergo [3], which provides a polymorphic type system). With such a prover as the verification back end, the expressions from the richer language have to be translated into the simpler logic. We describe two approaches to this translation in Section 4 one that captures type information using logical guards and one that encodes type parameters of polymorphic functions as additional function arguments. In both cases, it is necessary to encode 
Boogie's types as terms (so that typing conditions can be expressed as formulae), which is the subject of this section.

As a simply typed target language, we use a subset of the Boogie expression language, restricting the available types to (i) the built-in types bool and int (other types supported directly by the simply typed logic can be treated analogously to int), (ii) a type $U$ for (non-bool, non-int) individuals, and (iii) a type $T$ for (encoded) types. If necessary, expressions in this simply-typed language could be translated further to an untyped logic by adding domain predicates and guards for the types bool, int, $U$, and $T$. Because current SMT solvers are able to directly handle the four types, however, such a translation will usually not be required. Furthermore, we introduce a function symbol type $: U \rightarrow T$ that maps individuals to their type.

We encode types so that $T$ forms an algebraic datatype. If the target logic has direct support for algebraic datatypes, one may be able to build on it; in the scope of this paper, we use functions and axioms to describe the encoding.

\subsection{Type Constructors}

Each type constructor $C \in \mathcal{C}$ gives rise to a function symbol $C^{\#}: T^{\operatorname{arity}(C)} \rightarrow T$, as well as an axiomatization of a number of properties, including distinctness and injectivity. To formalize that the images of different type-constructor functions $C^{\#}$ are disjoint, we introduce a function Ctor: $T \rightarrow$ int and, for each type constructor $C$, a unique constant $n_{C}$. Injectivity is achieved by defining selector functions $C^{-1}, \ldots, C^{-n}: T \rightarrow T$ for each $n$-ary type constructor $C:$

$$
\left(\forall \bar{x}: T \bullet \operatorname{Ctor}\left(C^{\#}(\bar{x})\right)=n_{C}\right) \wedge \bigwedge_{i=1}^{\operatorname{arity}(C)}\left(\forall \bar{x}: T \bullet C^{-i}\left(C^{\#}(\bar{x})\right)=x_{i}\right)
$$

Theoretically, further axioms are needed for a faithful model of the type system. However, because these additional axioms are of a kind that cannot be expected to be useful for SMT solvers (e.g., statements about well-foundedness), we practically use only the axioms shown above in the Boogie implementation.

\subsection{Reduction of Map Types to Ordinary Type Constructors}

The encoding of Boogie's polymorphic map types is done by a reduction to normal type constructors: a map type $t$ containing the free type variables $\alpha_{1}, \ldots, \alpha_{n}$ (and arbitrary bound variables) can be encoded like a type expression $C_{t} \alpha_{1}, \ldots, \alpha_{n}$, for some fresh constructor $C_{t}$. The access functions can then be seen and axiomatized as ordinary functions select $_{t}$, store ${ }_{t}$, based on the axioms of the first-order theory of arrays [19].

There is a caveat in this construction: if two map types $s, t$ have common instances $u=\sigma_{s}(s)=\sigma_{t}(t)$, then an encoding of $u$ using either $C_{s}$ or $C_{t}$ will be overly restrictive. In particular, it might happen that $u$ is encoded as $C_{s}$ in one part, and as $C_{t}$ in another part of the same formula, leading to incompleteness:

function $f\langle\alpha\rangle(\alpha)$ returns (int); axiom $(\forall\langle\alpha\rangle m:[\alpha]$ int $\bullet f(m)=0)$; axiom $(\forall\langle\alpha\rangle m$ : [int $] \alpha \bullet f(m)=1)$; 
If $s=[\alpha]$ int in the first axiom happens to be encoded as $C_{s} \alpha$, and $t=[$ int $] \alpha$ in the second axiom as $C_{t} \alpha$, then the inconsistency of the two axioms will be lost: $C_{s} \alpha$ and $C_{t} \alpha$ do not have any common instances. The solution is to define larger classes of type constructors for map types: we abstract over map types and define constructors only for "most general" map types. Let us be more precise.

Given two types $s, t \in$ Type, we write $t \sqsubseteq s$ and say that $t$ is an instance of $s$ iff there is a substitution $\sigma$ such that $\sigma(s) \equiv t$. Observe that $\sqsubseteq$ is a pre-order on types, but not a partial order because anti-symmetry is violated for types that differ only in the names of free variables. The induced equivalence relation is denoted with $\cong$ : for $s, t \in$ Type, we define $s \cong t$ iff $s \sqsubseteq t$ and $t \sqsubseteq s$. It is the case that $\equiv \subseteq \cong$.

The pre-order $\sqsubseteq$ is canonically extended to Type $C=$ Type $/ \cong$ and partially orders the set. In fact, ( Type $C$, $)$ ) is a join-semi-lattice (i.e., any two types have a least common upper bound) whose $T$-element is the class of type variables $\alpha$. The strict order $\sqsubset$ satisfies the ascending chain condition (ACC): every ascending chain of types in Type $C$ eventually becomes stationary. This is important, because it justifies the existence of most-general map types that are the basis for our map-type encoding.

Let $\mathcal{M}_{C} \subseteq$ Type $C$ be the set of $\sqsubseteq$-maximal type classes whose elements start with the map type constructor, and let $\mathcal{M}$ be a set of unique representatives for all classes in $\mathcal{M}_{C}$. The elements of $\mathcal{M}$ can be seen as skeletons of map types and determine the binding and occurrences of bound type variables. Examples of types in $\mathcal{M}$ are:

$$
[\alpha] \beta \quad[\alpha, \beta] \gamma \quad[\alpha, \beta, \gamma] \delta \quad\langle\alpha\rangle[\alpha] \alpha \quad\langle\alpha\rangle[\alpha] \beta \quad\langle\alpha\rangle[\alpha](C \alpha)
$$

For every type $t$ that starts with a map type constructor, there is a unique type $m=$ $\operatorname{skel}(t) \in \mathcal{M}$ with $t \sqsubseteq m$. For example, skel $(\langle\alpha\rangle[C \alpha$, int $]$ bool $)=\langle\alpha\rangle[C \alpha, \beta] \gamma$. This means that every map type $t$ (also types containing free variables) can be represented in the form $\sigma(\operatorname{skel}(t))$, whereby the substitution $\sigma$ is uniquely determined for all variables that occur free in $\operatorname{skel}(t)$. We write flesh $(t)$ for the unique substitution satisfying flesh $(t)(\operatorname{skel}(t))=t$ whose domain is a subset of $\left\{\alpha_{1}, \ldots, \alpha_{n}\right\}$, where $\alpha_{1}, \ldots, \alpha_{n}$ are the free variables in $\operatorname{skel}(t)$. For example, flesh $(\langle\alpha\rangle[C \alpha$, int $]$ bool $)=$ $(\beta \mapsto$ int, $\gamma \mapsto$ bool $)$.

Translation of Types to Terms. In order to encode types, for each type $t \in \mathcal{M}$ that contains $n$ free type variables $\alpha_{1}, \ldots, \alpha_{n}$, we introduce a new $n$-ary function symbol $\mathbf{M}_{t}^{\#}: T^{n} \rightarrow T$. We will use the notation $\operatorname{Skel}^{\#}(s):=\mathrm{M}_{\text {skel }(s)}^{\#}$ for the skeleton symbol of an arbitrary map type $s$, and $\operatorname{Skel}^{-i}(s):=\mathrm{M}_{\text {skel }(s)}^{-i}$ for the selectors. Given an instantiation $\mu: \mathcal{A} \rightarrow$ Term of type variables, types can then be translated to terms:

$$
\begin{gathered}
\llbracket \alpha \rrbracket_{\mu}=\mu(\alpha) \quad \llbracket C t_{1} \ldots t_{n} \rrbracket_{\mu}=C^{\#}\left(\llbracket t_{1} \rrbracket_{\mu}, \ldots, \llbracket t_{n} \rrbracket_{\mu}\right) \\
\llbracket m \rrbracket_{\mu}=\operatorname{Skel}^{\#}(m)\left(\llbracket f \operatorname{lesh}(m)\left(\beta_{1}\right) \rrbracket_{\mu}, \ldots, \llbracket f \operatorname{llesh}(m)\left(\beta_{n}\right) \rrbracket_{\mu}\right)
\end{gathered}
$$

In the last equation, $m$ is a map type $\langle\bar{\alpha}\rangle[\bar{s}] t$ such that $\operatorname{skel}(m)$ contains the free type variables $\beta_{1}, \ldots, \beta_{n}$ (in this order of occurrence). Some examples are:

$$
\begin{aligned}
\llbracket C T \rrbracket_{\mu} & =C^{\#}\left(T^{\#}\right) & \llbracket[\text { int }] T \rrbracket_{\mu} & =\mathrm{M}_{[\alpha] \beta}^{\#}\left(i n t^{\#}, T^{\#}\right) \\
\llbracket[T] S \rrbracket_{\mu} & =\mathbf{M}_{[\alpha] \beta}^{\#}\left(T^{\#}, S^{\#}\right) & \llbracket\langle\alpha\rangle[\alpha] S \rrbracket_{\mu} & =\mathbf{M}_{\langle\alpha\rangle[\alpha] \beta}^{\#}\left(S^{\#}\right)
\end{aligned}
$$


Symbols and Axioms of Maps with Map Reduction. The access functions select and store can be seen and axiomatized as ordinary functions, based on the axioms of the first-order theory of arrays [19]. For each map type $m \in \mathcal{M}$, we introduce separate

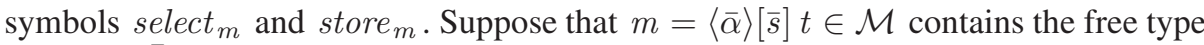
variables $\bar{\beta}=\left(\beta_{1}, \ldots, \beta_{n}\right)$ (in this order of occurrence). Then, the access functions have the following types:

$$
\text { select }_{m}\langle\bar{\alpha}, \bar{\beta}\rangle(m, \bar{s}) \text { returns }(t) \quad \text { store }_{m}\langle\bar{\alpha}, \bar{\beta}\rangle(m, \bar{s}, t) \text { returns }(m)
$$

It is necessary to include both $\bar{\alpha}$ and $\bar{\beta}$ as type parameters, because $m$ is parametric in the latter, and $\bar{s}$ and $t$ might be parametric in both. The semantics of maps is defined by axioms similar to the standard axioms of non-extensional arrays [19] ( $\bar{\alpha}^{\prime}$ is a vector of fresh type variables, and $\bar{\alpha} \mapsto \bar{\alpha}^{\prime}$ the substitution that replaces $\bar{\alpha}$ with $\bar{\alpha}^{\prime}$ ):

$$
\begin{gathered}
\left(\forall\langle\bar{\alpha}, \bar{\beta}\rangle h: m, \bar{x}: \bar{s}, z: t \bullet \operatorname{select}_{m}\left(\text { store }_{m}(h, \bar{x}, z), \bar{x}\right)=z\right) \wedge \\
\left(\forall\left\langle\bar{\alpha}, \bar{\alpha}^{\prime}, \bar{\beta}\right\rangle h: m, \bar{x}: \bar{s}, \bar{y}:\left(\bar{\alpha} \mapsto \bar{\alpha}^{\prime}\right) \bar{s}, z: t \bullet\right. \\
\left.\bar{x}=\bar{y} \vee \operatorname{select}_{m}\left(\text { store }_{m}(h, \bar{x}, z), \bar{y}\right)=\operatorname{select}_{m}(h, \bar{y})\right)
\end{gathered}
$$

\section{Translation of Expressions}

We define two main approaches to translating typed Boogie expressions into equivalent simply typed expressions: one that captures type information using logical guards (Section 4.1) and one that encodes type parameters of polymorphic functions as ordinary (additional) arguments (Section 4.2). The second encoding relies on the usage of e-matching to instantiate quantifiers (in contrast to methods like superposition used in first-order theorem provers), because typing information is generated such that triggers can only match on expressions of the right type (also see [6]).

The following Boogie program is used as running example for the translations:

function $\operatorname{Mojo}\langle\alpha\rangle(\alpha)$ returns (int); axiom $(\forall x:$ int $\bullet \operatorname{Mojo}(x)=x)$; type GuitarPlayer; $\quad \operatorname{axiom}(\forall g:$ GuitarPlayer $\bullet \operatorname{Mojo}(g)=68)$;

Note that it is essential to take the types of the quantified variables into account to not introduce inconsistent axioms.

\subsection{Translation Using Type Guards}

There is a long tradition of encoding type information using type guards, e.g., [17/677. As this translation is rather naive and has the disadvantage of complicating the propositional structure of formulae, it has been claimed [6] that its performance impact is prohibitive for many applications. We are able to show in Section 5, however, that this is no longer the case with state-of-the-art SMT solvers.

The Mojo example is complemented with type guards as follows. Because the quantified formulae are now guarded and only concern individuals of the right types, no contradiction is introduced. The function $i 2 u$ is defined below.

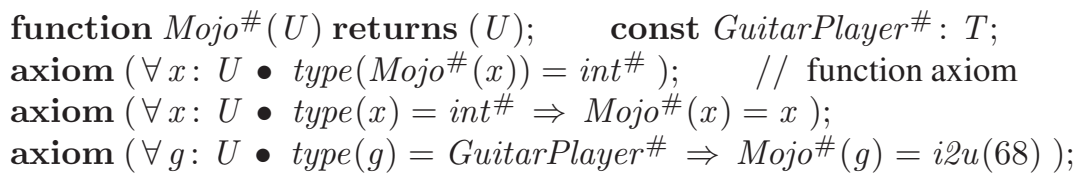


Function Axioms. During the translation, user-defined Boogie functions are replaced with $U$-typed functions. For a function $f\left\langle\alpha_{1}, \ldots, \alpha_{m}\right\rangle\left(s_{1}, \ldots, s_{n}\right)$ returns $(t)$ such that $\alpha_{1}, \ldots, \alpha_{k}$ do not occur in $s_{1}, \ldots, s_{n}$ (but only in $t$ ), while $\alpha_{k+1}, \ldots, \alpha_{m}$ occur in $s_{1}, \ldots, s_{n}$ (and possibly in $t$ ), this post-translation function $f^{\#}$ has the type $T^{k} \times U^{n} \rightarrow U$. We will capture the original typing with an axiom of the shape:

$$
\left(\forall \bar{x}: \bar{U}, \bar{y}: \bar{T} \bullet \operatorname{type}\left(f^{\#}(\bar{y}, \bar{x})\right)=\llbracket t \rrbracket_{\mu}\right)
$$

This axiom does not contain any quantifiers corresponding to $\alpha_{k+1}, \ldots, \alpha_{m}$ that occur in $s_{1}, \ldots, s_{n}$, which is advantageous for SMT solvers because the formula does not offer good triggers for $\alpha_{k+1}, \ldots, \alpha_{m}$. Instead, the mapping $\mu: \mathcal{A} \rightarrow$ Term that determines the values of type parameters plays a prominent role. We define this mapping using extractor terms, which are recursively defined over types and describe how the type parameter values can be reconstructed from the actual arguments $\bar{x}$ with the help of the selector functions $C^{-i}$ defined in Section 3.1

Suppose that $\alpha \in \mathcal{A}$ is a type variable. Assuming that the term $E$ encodes the type $t \in$ Type, the set extractors $\alpha(E, t)$ specifies terms that compute $\alpha$ 's value:

$$
\begin{aligned}
\text { extractors }_{\alpha}(E, \beta) & =\text { if } \alpha=\beta \text { then }\{E\} \text { else } \emptyset \\
\text { extractors }_{\alpha}\left(E, C t_{1} \ldots t_{n}\right) & =\bigcup_{i=1}^{n} \text { extractors }_{\alpha}\left(C^{-i}(E), t_{i}\right) \quad(C \in C) \\
\text { extractors }_{\alpha}(E, m) & =\bigcup_{i=1}^{n} \text { extractors }_{\alpha}\left(\operatorname{Skel}^{-i}(m)(E), \operatorname{flesh}(m)\left(\gamma_{i}\right)\right)
\end{aligned}
$$

In the last equation, $m$ is a map type $\langle\bar{\beta}\rangle[\bar{s}] t$ such that $\operatorname{skel}(m)$ contains the free type variables $\gamma_{1}, \ldots, \gamma_{n}$ (in this order of occurrence). Some examples are:

$$
\begin{aligned}
\text { extractors }_{\alpha}(x, C \beta \alpha) & =\left\{C^{-2}(x)\right\} \\
\text { extractors }_{\alpha}(x,\langle\beta\rangle[C \beta \alpha] \alpha) & =\left\{C^{-2}\left(\mathrm{M}_{\langle\beta\rangle[C \beta \gamma] \delta}^{-1}(x)\right), \mathrm{M}_{\langle\beta\rangle[C \beta \gamma] \delta}^{-2}(x)\right\}
\end{aligned}
$$

The extractor $C^{-2}(x)$, for instance, can derive $\alpha$ 's value from the instance $C$ int bool of $C \beta \alpha$, resulting in $C^{-2}(\llbracket C$ int bool $\rrbracket)=C^{-2}\left(C^{\#}\left(\right.\right.$ int $^{\#}$, bool $\left.\left.{ }^{\#}\right)\right)=$ bool $^{\#}$.

A simple optimization (that is implemented in Boogie but left out from this paper for reasons of presentation) is to keep argument or result types int and bool of functions, instead of replacing them with $U$. This can reduce the number of casts to and from $U$ later needed in the translation.

Embedding of Built-in Types. SMT solvers offer built-in types like booleans, integers, and bit vectors, whose usage is crucial for performance. We define casts to and from the type $U$ in order to integrate built-in types into our framework. For the built-in types bool and int, we introduce the cast functions $i 2 u:$ int $\rightarrow U, u 2 i: U \rightarrow$ int, b2u $:$ bool $\rightarrow U, u 2 b: U \rightarrow$ bool and axiomatize them as:

$$
\begin{aligned}
& \left(\forall x: \text { int } \bullet \operatorname{type}(i 2 u(x))=i n t^{\#} \wedge u 2 i(i 2 u(x))=x\right) \wedge \\
& \left(\forall x: U \bullet \operatorname{type}(x)=i n t^{\#} \Rightarrow i 2 u(u 2 i(x))=x\right)
\end{aligned}
$$

and analogously for bool. The axioms imply that $i 2 u$ and $b 2 u$ are embeddings into $U$, and that $u 2 i$ and $u 2 b$ are their inverses. For simplicity, in the following translation we insert casts in each place where operators over bool or int occur, although many of the casts could directly be eliminated using the axioms. Such optimizations are present in the Boogie implementation as well. 
Translation of Expressions. Given an instantiation $\mu: \mathcal{A} \rightarrow$ Term of type variables, the main cases of the translation are:

$$
\begin{aligned}
& \llbracket x \rrbracket_{\mu}=x \\
& \llbracket f\left(E_{1}, \ldots, E_{n}\right) \rrbracket_{\mu}=f^{\#}\left(\llbracket E_{1} \rrbracket_{\mu}, \ldots, \llbracket E_{n} \rrbracket_{\mu}\right) \\
& \llbracket E=F \rrbracket_{\mu}=b 2 u\left(\llbracket E \rrbracket_{\mu}=\llbracket F \rrbracket_{\mu}\right) \\
& \llbracket E+F \rrbracket_{\mu}=i 2 u\left(u \mathscr{2 i}\left(\llbracket E \rrbracket_{\mu}\right)+u 2 i\left(\llbracket F \rrbracket_{\mu}\right)\right) \\
& \llbracket E \wedge F \rrbracket_{\mu}=b 2 u\left(u 2 b\left(\llbracket E \rrbracket_{\mu}\right) \wedge u 2 b\left(\llbracket F \rrbracket_{\mu}\right)\right) \\
& \llbracket(\forall\langle\bar{\alpha}\rangle \bar{x}: \bar{t} \bullet E) \rrbracket_{\mu}=b 2 u\left(\forall \bar{x}: \bar{U}, \bar{y}: \bar{T} \bullet \operatorname{type}(\bar{x})=\llbracket \bar{t} \rrbracket_{\mu^{\prime}} \Rightarrow u 2 b\left(\llbracket E \rrbracket_{\mu^{\prime}}\right)\right) \\
& \llbracket(\exists\langle\bar{\alpha}\rangle \bar{x}: \bar{t} \bullet E) \rrbracket_{\mu}=b 2 u\left(\exists \bar{x}: \bar{U}, \bar{y}: \bar{T} \bullet \operatorname{type}(\bar{x})=\llbracket \bar{t} \rrbracket_{\mu^{\prime}} \wedge u 2 b\left(\llbracket E \rrbracket_{\mu^{\prime}}\right)\right)
\end{aligned}
$$

In the last two equations, $\bar{y}$ is a vector of fresh variables, and $\mu^{\prime}=(\mu, \bar{\alpha} \mapsto \bar{y})$. In the case that a type parameter $\alpha_{i}$ occurs in some of the types $\bar{t}$, a more efficient translation is possible by extracting the value of $\alpha_{i}$ from the bound variables $\bar{x}$ :

$$
\mu^{\prime}\left(\alpha_{i}\right) \in \bigcup_{j=1}^{m} \text { extractors }_{\alpha_{i}}\left(\text { type }\left(x_{j}\right), t_{j}\right)
$$

The optimization is particularly relevant with e-matching-based SMT solvers, because the formula resulting from the original translation often does not contain good triggers for the variables $\bar{y}$ : type parameters $\bar{\alpha}$ are used only in types, which usually do not provide good discrimination for instantiation.

\subsection{Translation Using Type Arguments}

Our second translation works by explicitly passing the values of type parameters to functions. In the context of SMT solvers, this allows us to completely leave out type guards and leads to formulae with a simpler propositional structure, albeit functions have a higher arity and more terms occur in the formulae. It has to be noted that this second translation crucially depends on the usage of an SMT solver with e-matching: such solvers are not able to exploit missing type guards, because typing information is inserted in expressions in such a way that triggers can only match on expressions of the right type. The translation trades generality for performance: while it is not applicable with most first-order theorem provers (e.g., superposition provers), the experimental evaluation in Section 5 shows a clear performance gain compared to the type guard translation from the previous section. A similar observation is made in [6].

When using type arguments, the Mojo example gets translated as follows:

$$
\begin{aligned}
& \text { function } \operatorname{Mojo}^{\#}(T, U) \text { returns }(U) ; \quad \operatorname{axiom}\left(\forall x: U \bullet \operatorname{Mojo}^{\#}\left(\text { int }^{\#}, x\right)=x\right) \text {; } \\
& \text { const GuitarPlayer }
\end{aligned}
$$

The Typing of Functions. A function $f\left\langle\alpha_{1}, \ldots, \alpha_{m}\right\rangle\left(s_{1}, \ldots, s_{n}\right)$ returns $(t) \in \mathcal{F}$ is during the translation replaced by a function $f^{\#}$ with the type $T^{m} \times U^{n} \rightarrow U$, i.e., the type parameters are given the status of ordinary function arguments. It is unnecessary to generate typing axioms for $f^{\#}$, since typing information is inserted everywhere in terms during the translation and does not have to be derived by the SMT solver. 


\begin{tabular}{|c|c|c|c|}
\hline & Type Guards & Type Arguments & No Types \\
\hline \multicolumn{4}{|c|}{ Z3 2.0 } \\
\hline Boogie & $2002 / 595 / 1,0.781 \mathrm{~s}$ & $2000 / 597 / 1,0.651 \mathrm{~s}$ & $1984 / 613 / 1,0.813 \mathrm{~s}$ \\
\hline$(7840)$ & $6999 / 839 / 2,3.447 \mathrm{~s}$ & $6999 / 836 / 5,2.181 \mathrm{~s}$ & $6999 / 836 / 5,2.196 \mathrm{~s}$ \\
\hline HAVOC (385) & $353 / 16 / 16, \quad 0.709 \mathrm{~s}$ & $351 / 18 / 16, \quad 0.524 \mathrm{~s}$ & $350 / 17 / 18, \quad 0.367 \mathrm{~s}$ \\
\hline \multicolumn{4}{|c|}{ Z3 1.3 } \\
\hline Boogie & $1978 / 609 / 3 \quad 1.107$ & $1974 / 611 / 5 \quad 1.212$ & $1961 / 626 / 3 \quad 2.385$ \\
\hline
\end{tabular}

Fig. 5. Results for the different benchmark categories. In each cell, we give the number of times the outcome valid/invalid/timeout occurred, as well as the average time needed for successful proof attempts (i.e., counting cases with the outcome valid or invalid).

Translation of Expressions. We maintain both an instantiation $\mu: \mathcal{A} \rightarrow$ Term and an environment $\mathcal{V}: \mathcal{X} \rightarrow$ Type that assigns types to variables during the translation:

$$
\begin{aligned}
\llbracket x \rrbracket_{\mu, \mathcal{V}} & =x \\
\llbracket f(\bar{E}) \rrbracket_{\mu, \mathcal{V}} & =f^{\#}\left(\llbracket \sigma(\bar{\alpha}) \rrbracket_{\mu, \mathcal{V}}, \llbracket \bar{E} \rrbracket_{\mu, \mathcal{V}}\right) \\
\llbracket E=F \rrbracket_{\mu, \mathcal{V}} & =b 2 u\left(\llbracket E \rrbracket_{\mu, \mathcal{V}}=\llbracket F \rrbracket_{\mu, \mathcal{V}} \wedge \llbracket t_{E} \rrbracket_{\mu}=\llbracket t_{F} \rrbracket_{\mu}\right) \\
\llbracket E+F \rrbracket_{\mu, \mathcal{V}} & =i 2 u\left(u 2 i\left(\llbracket E \rrbracket_{\mu, \mathcal{V}}\right)+u 2 i\left(\llbracket F \rrbracket_{\mu, \mathcal{V}}\right)\right) \quad \ldots \\
\llbracket E \wedge F \rrbracket_{\mu, \mathcal{V}} & =b 2 u\left(u 2 b\left(\llbracket E \rrbracket_{\mu, \mathcal{V}}\right) \wedge u 2 b\left(\llbracket F \rrbracket_{\mu, \mathcal{V}}\right)\right) \quad \ldots \\
\llbracket(Q\langle\bar{\alpha}\rangle \bar{x}: \bar{t} \bullet E) \rrbracket_{\mu, \mathcal{V}} & =b 2 u\left(Q \bar{x}: \bar{U}, \bar{y}: \bar{T} \bullet u 2 b\left(\llbracket E \rrbracket_{(\mu, \bar{\alpha} \mapsto \bar{y}),(\mathcal{V}, \bar{x} \mapsto \bar{t})}\right)\right)
\end{aligned}
$$

The second equation assumes $f$ has typing $\langle\bar{\alpha}\rangle(\bar{s})$ returns $(t)$ and that $\sigma$ is the instantiation of the type parameters $\bar{\alpha}$ that is inferred when applying $f$ to $\bar{E}$. The types $t_{E}, t_{F}$ in the third equation are determined by $\mathcal{V} \Vdash E: t_{E}$ and $\mathcal{V} \Vdash F: t_{F}$. In the last equation, $\bar{y}$ is a vector of fresh variables, and $Q \in\{\forall, \exists\}$ is a quantifier.

\section{Experimental Results and Related Work}

We quantitatively evaluate the two different translations of Boogie expressions, together with a third unsound translation that simply erases all type information. The third translation is close to the translation used by the Boogie 1 tool, so that a comparison between Boogie 2 and Boogie 1 is possible. The evaluated Boogie programs are:

- The Boogie and SscBoogie regression test suites: A collection of correct and incorrect programs written in Boogie, Spec\# [1], and Dafny [14] that make use of polymorphism; also parts of the Boogie tool itself (a Spec\# program) are included.

- Hyper-V verification conditions generated by VCC [5]: Boogie programs that stem from a project to verify the Microsoft hypervisor Hyper-V.

- Benchmarks from the HAVOC tool [4]: Regression tests and verification conditions to prove memory safety and invariants of various $\mathrm{C}$ programs.

Because the programs of the last two categories do not use polymorphism, the overhead of our translations for simple problems (that could really be handled with the "No Types" translation) is measured. 
For each of the categories, we used Boogie 2 to generate verification conditions with the different translations and write them to separate files. We then measured the performance of the state-of-the-art SMT solver Z3 2.01 on the altogether more than 10,000 verification conditions. The prover was run on each verification condition with a timeout of 120s (1800s for the Boogie tests), measuring the average time needed over three runs. All experiments were made on an Intel Core 2 Duo, 3.16GHz, with 4GB.

Figure 5 summarizes the results. The time difference between the type argument encoding and the translation without types is always very small, the argument encoding is even faster in two categories. The type guard encoding is close to the other translations on the Boogie tests, but is on average about 55\% slower on the VCC examples, and performs similarly on the HAVOC examples. One explanation for this phenomenon is that (in particular) VCC generates a large number of Boogie functions, which leads to a large number of additional axioms in the type guard encoding.

Related Work. The intermediate verification language Boogie is most closely related to Why [10], which offers ML-style polymorphism [22]. ML-style polymorphism (or "let polymorphism") is more limited than the higher-rank polymorphism in Boogie; for example, it does not allow polymorphic map types nor general quantifications over types, both of which are used heavily by some Boogie front ends. Our typing rule for equality is similar to the "heterogeneous equality" introduced in [18]. Meanwhile, compilers have also explored the benefits of using typed intermediate languages [21].

Couchot and Lescuyer turn formulae with ML-style polymorphism into multi-sorted and untyped formulae [6], taking advantage of built-in theories. They have implemented their translations as modules of the Why tool [10] and report on some experiments. With Simplify [9], they measure a $200 \%$ slowdown with their version of a type guard translation, and a $300 \%$ slowdown with their other encoding (which is somewhat similar to our type argument encoding). In contrast, we measure a slowdown of at most $95 \%$ with the type guards encoding and at most $45 \%$ with the type arguments encoding.

Bobot et al. show how to incorporate ML-style polymorphism directly into an SMT solver [3]. Our type arguments translation is quite similar to the machinery they present. It would be interesting to put to test their conjecture that building polymorphism into a prover is a better solution than handling it through a pre-processing step.

There is a large body of work on the encoding of (typed) higher-order logic (HOL) in first-order logic (FOL). Such translations primarily target FOL provers, in contrast to SMT solvers as in our case. Meng and Paulson [20] enrich terms with type annotations in the form of first-order functions and describe different translations, some of which are sound, while others require proofs to be typechecked and possibly rejected afterwards. Similarly, Hurd [12] describes translations from HOL to FOL in which type information can be included in the operator for function application, which is similar to our type argument encoding (and in particular the handling of map types). Translations in the same spirit as our type guard encoding have been studied [7] for the Mizar language.

\section{Conclusions}

We have introduced the type system of Boogie 2, shown how its advanced type features are useful to program verifiers in encoding program semantics, and shown how to

\footnotetext{
1 http: / / research.microsoft.com/projects/z3/
} 
translate its polymorphic types and expressions into first-order formulae suitable for SMT solvers. Our experimental data support the idea that including such advanced features in an intermediate verification language is both desirable for verifier front ends and feasible for performance. Future work include further optimizations like monomorphization.

Acknowledgments. We thank Stephan Tobies and Shuvendu Lahiri for providing us with Boogie files from VCC and HAVOC for use as benchmarks. We also thank Michał Moskal, Nikolaj Bjørner, and Leonardo de Moura for help with Z3, and them, Alastair Donaldson, Shaz Qadeer, Andrei Voronkov for useful discussions and comments. Finally, we are grateful for valuable comments from the anonymous referees.

\section{References}

1. Barnett, M., Chang, B.Y.E., DeLine, R., Jacobs, B., Leino, K.R.M.: Boogie: A modular reusable verifier for object-oriented programs. In: de Boer, F.S., Bonsangue, M.M., Graf, S., de Roever, W.-P. (eds.) FMCO 2005. LNCS, vol. 4111, pp. 364-387. Springer, Heidelberg (2006)

2. Barrett, C., Ranise, S., Stump, A., Tinelli, C.: The Satisfiability Modulo Theories Library, SMT-LIB (2008), WWW . SMT-LIB . org

3. Bobot, F., Conchon, S., Contejean, E., Lescuyer, S.: Implementing polymorphism in SMT solvers. In: SMT 2008 (2008)

4. Chatterjee, S., Lahiri, S.K., Qadeer, S., Rakamarić, Z.: A reachability predicate for analyzing low-level software. In: Grumberg, O., Huth, M. (eds.) TACAS 2007. LNCS, vol. 4424, pp. 19-33. Springer, Heidelberg (2007)

5. Cohen, E., Moskal, M., Schulte, W., Tobies, S.: A practical verification methodology for concurrent programs. MSR-TR 2009-15, Microsoft Research (2009)

6. Couchot, J.F., Lescuyer, S.: Handling polymorphism in automated deduction. In: CADE-21, pp. 263-278 (2007)

7. Dahn, I.: Interpretation of a Mizar-like logic in first-order logic. In: Caferra, R., Salzer, G. (eds.) FTP 1998. LNCS (LNAI), vol. 1761, pp. 137-151. Springer, Heidelberg (2000)

8. DeLine, R., Leino, K.R.M.: BoogiePL: A typed procedural language for checking objectoriented programs. MSR-TR 2005-70, Microsoft Research (March 2005)

9. Detlefs, D., Nelson, G., Saxe, J.B.: Simplify: a theorem prover for program checking. J. ACM 52(3), 365-473 (2005)

10. Filliâtre, J.C.: Why: a multi-language multi-prover verification tool. Research Report 1366, LRI, Université Paris Sud (March 2003)

11. Flanagan, C., Leino, K.R.M., Lillibridge, M., Nelson, G., Saxe, J.B., Stata, R.: Extended static checking for Java. In: PLDI 2002. ACM, New York (2002)

12. Hurd, J.: First-order proof tactics in higher-order logic theorem provers. Technical Report NASA/CP-2003-212448, pp. 56-68 (2003)

13. Leino, K.R.M.: This is Boogie 2. Manuscript KRML 178 (2008), http://research.microsoft.com/ leino/papers.html

14. Leino, K.R.M.: Specification and verification of object-oriented software. In: Summer School Marktoberdorf 2008. NATO ASI Series F. IOS Press, Amsterdam (2009)

15. Leino, K.R.M., Monahan, R.: Reasoning about comprehensions with first-order SMT solvers. In: SAC 2009, pp. 615-622. ACM, New York (2009)

16. Leino, K.R.M., Saxe, J.B., Stata, R.: Checking Java programs via guarded commands. FTfJP 1999. Tech. Rep. 251, Fernuniversität Hagen (May 1999) 
17. Manzano, M.: Extensions of First-Order Logic. Cambridge Tracts in Theoretical Computer Science. Cambridge University Press, Cambridge (1996)

18. McBride, C.: Elimination with a motive. In: Callaghan, P., Luo, Z., McKinna, J., Pollack, R. (eds.) TYPES 2000. LNCS, vol. 2277, pp. 197-216. Springer, Heidelberg (2002)

19. McCarthy, J.: Towards a mathematical science of computation. In: IFIP Congress 62, pp. 21-28. North-Holland, Amsterdam (1962)

20. Meng, J., Paulson, L.C.: Translating higher-order clauses to first-order clauses. J. Autom. Reason. 40(1), 35-60 (2008)

21. Morrisett, G., Walker, D., Crary, K., Glew, N.: From System F to typed assembly language. TOPLAS 21(3), 527-568 (1999)

22. Pierce, B.C.: Types and Programming Languages. The MIT Press, Cambridge (2002)

23. Poetzsch-Heffter, A.: Specification and verification of object-oriented programs. Habilitationsschrift, Technische Universität München (1997) 